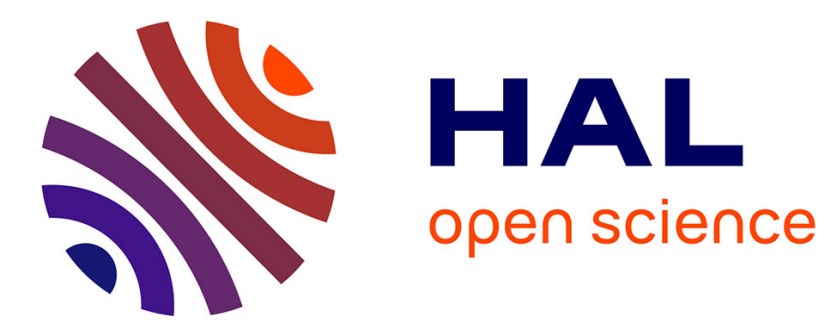

\title{
Art contemporain et territoires urbains: le musée MADRe à Naples
}

\author{
Pascale Froment
}

\section{To cite this version:}

Pascale Froment. Art contemporain et territoires urbains: le musée MADRe à Naples. Méditerranée, 2010, Villes culturelles en Méditerranée, 114, pp.103-112. 10.4000/mediterranee.4430 . hal-03561067

\section{HAL Id: hal-03561067 \\ https://hal.science/hal-03561067}

Submitted on 8 Feb 2022

HAL is a multi-disciplinary open access archive for the deposit and dissemination of scientific research documents, whether they are published or not. The documents may come from teaching and research institutions in France or abroad, or from public or private research centers.
L'archive ouverte pluridisciplinaire HAL, est destinée au dépôt et à la diffusion de documents scientifiques de niveau recherche, publiés ou non, émanant des établissements d'enseignement et de recherche français ou étrangers, des laboratoires publics ou privés. 


\title{
Art contemporain et territoires urbains : le musée MADRe à Naples
}

\author{
Contemporary art and urban territories: the MADRe Museum in Naples
}

\author{
Pascale FROMENT \\ Maître de conférences en géographie \\ UMR Telemme \\ MMSH Aix-en-Provence \\ frompasc@wanadoo.fr
}

\begin{abstract}
La création du musée MADRe en 2005 en plein coeur de Naples s'insère dans un parcours ambitieux de développement des lieux et manifestations de l'art contemporain dans la ville et de requalification des espaces dégradés du centre historique. La forme même du musée ainsi que le contexte économique et politique de sa naissance en font cependant un lieu paradoxal à bien des égards. Malgré des tentatives récentes de nouer le dialogue avec le territoire environnant à travers l'éducation à l'art, le MADRe demeure davantage tourné vers l'extérieur, obéissant à des logiques de renouvellement urbain bien connues et expérimentées dans de nombreuses métropoles européennes. En outre, les signes récurrents de contestation dont il est la cible symbolisent également les blocages croissants dans les relations entre institutions et société civile au détriment d'une véritable gouvernance.
\end{abstract}

Mots clés: Italie, Naples, cœur historique, art contemporain, culture, musée MADRe, régénération urbaine, gouvernance

La création en 2005 du musée d'art contemporain Donnaregina, ou musée MADRe, à Naples renvoie aux multiples facettes de la question du rapport entre art contemporain et territoires urbains, constamment reposée dans la mesure où depuis plus de trente ans se multiplient dans les métropoles européennes les lieux de l'art contemporain. Celui-ci, devenu en effet « un véritable secteur d'activité culturel, créateur d'emplois, de richesses et d'espaces » (GILABERT, 2004) s'impose de plus en plus comme moyen et comme enjeu d'autopromotion des villes dans la nouvelle concurrence des territoires à différentes échelles. La naissance du MADRe sous cet angle ne déroge guère à la règle qui s'est largement diffusée à partir des années 1990 dans les villes des pays de la rive nord méditerranéenne, accouchant de structures inégalement prestigieuses à Bilbao, Séville, Gênes ou Marseille...

Il n'est pas question toutefois dans le cadre de cette contribution de développer l'ensemble de ces aspects, mais plutôt d'interroger l'implantation de ce nouveau lieu au cour de Naples, métropole méridionale en pleine mutation, et rejoignant du même coup des pistes de réflexion plus amples quant au rôle de la fonction culturelle dans les politiques de renouvellement urbain.

La création du musée MADRe n'est pas une figure isolée, mais s'insère dans un contexte d'effervescence artistique et dans une stratégie ambitieuse de développement de l'art contemporain dans les politiques culturelles de la ville. Cette stratégie étroitement associée à une volonté de changement d'image de la métropole parthénopéenne et déployée sur quinze ans, obéit à des tempos, à des rythmes qu'il convient non seulement de dégager mais également de
The creation of the MADRe Museum in 2005 in the heart of Naples fits into an ambitious course of development of the places and events of contemporary art in the city and of recycling of degraded spaces in the historic core. The very form of this Museum, as well as the economic and political context of its birth, make it, however, a paradoxical place in many respects. In spite of recent attempts to initiate a dialog with the surrounding area through art education, the MADre still remains more oriented to foreign themes beyond the local in following a logic of well-known urban renewal in numerous European metropolitan centers. Furthermore, the recurrent challenges of which that museum is a target also symbolize the growing obstacles in the relations between institutions and civil society to the detriment of real governance.

Key words: Italy, Naples, historic core, contemporary art, culture, MADre Museum, urban renewal, governance

conjuguer à des fonctions et à des logiques spatiales qui ont elles-mêmes évolué. Depuis la «montagne de sel» de Mimmo Paladino sur la place du Plébiscite en 1995 aux stations de l'art qui scandent l'avancée du métro dans la ville et aux musées nouvellement créés, la pluralité des expressions urbaines de l'art contemporain à Naples qui jalonnent ce cheminement récent, nous invite à replacer l'avènement du MADRe dans une perspective plus large, à saisir sa spécificité, voire sa complémentarité avec les autres initiatives dans le même domaine, en particulier avec les stations de l'art.

La forme même de ce musée, la vocation qui lui est assignée renvoient en même temps à un problème d'articulation des espaces et des échelles. Il convient alors de s'interroger sur le sens à donner aux équipements culturels prestigieux comme le MADRe et sur le lien entre son insertion locale, dans le contexte napolitain, et son rayonnement à de plus vastes échelles: internationale dans un réseau de villes méditerranéennes toujours plus soucieuses de leur image et de leur positionnement européen, nationale compte tenu du climat politique et économique de la péninsule quelquefois entaché de sécessionnisme, enfin régionale et métropolitaine. De ce point de vue, la concentration d'œuvres très cotées sur le marché mondial de l'art contemporain, au sein de l'un des quartiers les plus déshérités de la ville, pose plus généralement le problème de la vocation culturelle assignée aux espaces en déprise, dans une perspective de développement local, qu'il s'agisse de vastes friches devenues des « hauts lieux » de la régénération urbaine ou, dans le cas qui nous occupe, de centres historiques désertés par leurs activités productives traditionnelles et minés par le chômage. 


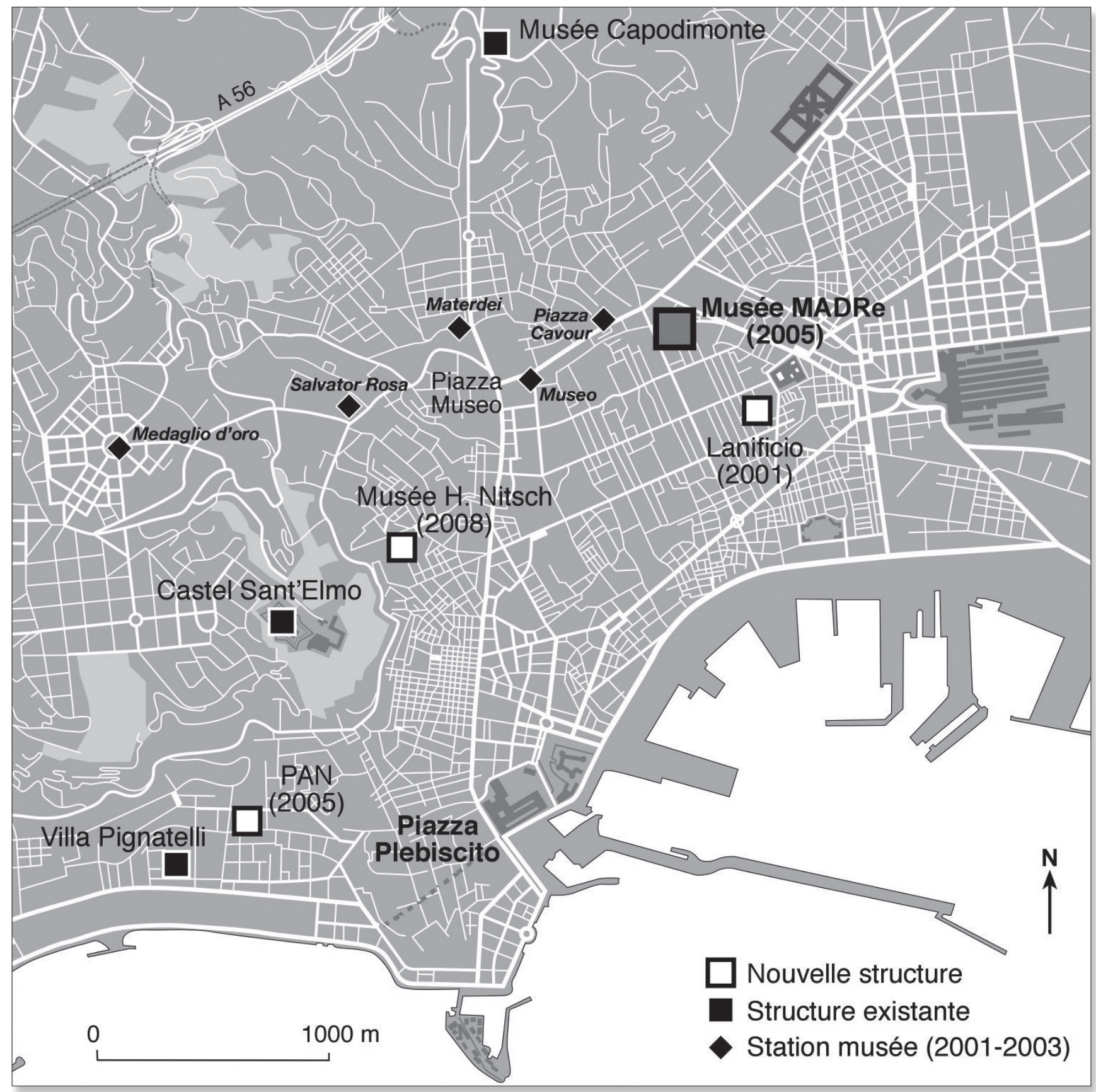

Fig. 1 - Principales structures et lieux liés à l'art contemporain.

Enfin, ces interrogations rejoignent celle des logiques des politiques de renouvellement urbain et de gouvernance à Naples, et de leur infléchissement depuis le début du millénaire qui correspond aussi à une évolution du contexte politique et économique de la ville. On observe en effet un renversement de tendance depuis le moment de la « renaissance napolitaine ", état de grâce qui court de 1993, date de l'élection d'Antonio Bassolino à la Mairie de Naples, à 1998, jusqu'à l'effritement de la confiance et au désarroi qui lui succèdent et trouvent leur acmé dans la récente crise des ordures en 2008. À cet égard, le MADre, symbolise aussi les crispations et les blocages croissants entre institutions et société civile et fait l'objet de contestations récurrentes.

Après une mise en perspective de la création du musée dans le nouveau dispositif culturel napolitain et en relation avec d'autres expériences nord-méditerranéennes, nous aborderons les aspects paradoxaux de l'enchâssement territorial de cette nouvelle structure dans les quartiers dégradés du centre historique. Il s'agira enfin de voir en quoi le nouveau musée peut être lu comme emblématique de la fermeture du dialogue entre acteurs institutionnels et forces vives de la ville, signifiant peut-être le retour à des formes plus rigides de gouvernement urbain au détriment d'une véritable gouvernance.

\section{I - Art contemporain et air du temps}

L'ouverture du musée MADRe à Naples, peut être considérée par bien des aspects comme le point d'orgue, l'aboutissement d'un parcours visant l'affirmation de l'art contemporain dans la ville, initié après 1995 par la nouvelle équipe municipale, un évènement qu'il convient de mettre en perspective dans le temps et dans l'espace ainsi que dans le contexte plus large des politiques de renouvellement urbain. 


\section{I.I - Parcours, déclinaisons de l'art contemporain à Naples}

La présence de l'art contemporain est attestée à Naples bien avant le début des années 1990. On en trouve des témoignages aussi bien sous forme institutionnelle dans la conception du centre directionnel par Kenzo Tange, qui transforme la skyline de la ville dans les années 1980, que sous une forme plus alternative avec l'initiative de l'artiste Ernest-Pignon-Ernest, dont les nombreuses figures iconiques puisées dans l'imaginaire et les mythes de la ville s'affichent sur les murs des ruelles de Naples entre 1988 et 1995. Toutefois ces marques, durables ou éphémères, d'inspiration profondément diverse, demeurent relativement éclatées, et l'art contemporain se signale jusqu'à une période récente essentiellement par l'activité des réseaux de galeristes locaux. Parallèlement, les formes muséales qui caractérisent la ville demeurent classiques et en même temps très désuètes; elles sont associées à un très riche patrimoine archéologique d'une part et aux collections fécondes de peinture napolitaine des XVII et XVIII ${ }^{\mathrm{e}}$ siècles d'autre part.

Les politiques culturelles, qui se mettent en place au milieu de la décennie 1990, jouent à la fois sur la revalorisation du patrimoine existant et sur la création d'un nouveau patrimoine artistique contemporain. L'intérêt de cet itinéraire, en rupture avec les politiques précédentes, vient de l'alliance de différentes formes et relations à l'espace qui s'inscrivent dans les quartiers centraux de la ville: formes muséales créées dans des bâtiments initialement prévus pour une autre fonction mais réaménagés à cet effet, espaces publics comme lieux d'exposition, remodelés, voire créés, par des artistes contemporains ou encore espaces alternatifs.

La mue, qui s'opère progressivement en deux étapes, passe dans un premier temps par un re-toilettage et une réorganisation des musées existants, comme le musée de Capodimonte qui ouvre une section d'art moderne et contemporain en 1996 ou encore le musée archéologique national et le Castel Sant'Elmo accueillant des expositions temporaires. La Place du Plébiscite, espace public devenu emblématique du changement urbain et de la nouvelle équipe municipale, devient rapidement un lieu de référence de l'art contemporain par les installations qui s'y succèdent selon un rythme annuel depuis 1995 (fig. 1).

À partir des années 2000, un pas décisif est franchi avec l'ouverture des six premières stations-musées de la ligne 1 du métro, inaugurées entre 2001 et 2003 . Chaque station a ses propres caractéristiques architecturales, adaptées à la singularité des sites, et selon un éclectisme d'ensemble lié à une grande pluralité des artistes et des œuvres proposées. L'avènement de ces stations a été suivi de la création de trois nouveaux réceptacles entièrement dévolus à l'art contemporain qui voient le jour entre 2005 et 2009, au point que cer- tains parlent de « muséomanie ». Il est nécessaire toutefois de distinguer, au sein de ces nouvelles structures, entre d'une part le PAN ou Palazzo delle Arti di Napoli', ouvert en mars 2005 dans les quartiers chics de la ville, dont l'objectif encore assez mal dessiné en fait une coquille un peu creuse, le musée Hermann Nitsch, inauguré en septembre 2008, franchement autoréférentiel, et d'autre part le musée MADRe, véritable musée d'art contemporain ouvert en deux temps entre juin et décembre 2005. À côté de ces espaces investis sur un mode institutionnel, certains lieux voient le jour de façon plus alternative, comme le Lanificio $^{2}$, récupéré par de jeunes artistes en 2001 et transformé en lieu de rencontres artistiques en plein centre historique. Le début du millénaire marque donc un tournant et symbolise la volonté d'ancrer sur le long terme l'art contemporain dans les politiques de la ville.

\section{I.2 - Mimétismes}

Comme dans la plupart des métropoles mondiales, le recours croissant à l'art contemporain est lié à Naples à une politique d'image et de promotion de la ville qui s'inscrit dans une dynamique de renouvellement urbain initiée après 1993, avec le changement politique incarné par Antonio Bassolino. Ces politiques font la part belle à la requalification du centre historique, aux infrastructures de transport et aux équipements culturels, qui s'arrogent une place de choix dans la plupart des interventions d'aménagement urbain en Europe depuis trente ans. Comme ailleurs aussi, elles font partie de l'arsenal des réponses apportées à une situation de crise - crise financière et crise d'image, crise sociale et crise d'espaces tombés en déshérence -, pour lesquels la culture ${ }^{3}$ associée au tourisme devient une voie presque obligée.

De ce point de vue, Naples fait écho, avec quelque retard, aux partis pris et aux réalisations intervenues un peu partout sur la rive nord de la Méditerranée et qui allient étroitement trois ingrédients majeurs :

- la fonction culturelle, "garante de la redynamisation économique et sociale, de la construction d'une image urbaine forte » (RODRIGUES-MALTA, 2001) devient un pivot des nouvelles politiques urbaines en ce qu'elle participe de la construction de la " ville créative », de sa compétitivité (FLORIDA, 2002), vecteur de développement local et de sa visibilité internationale. L'offre culturelle, liée à la vocation touristique dévolue au centre historique, se déploie dans des directions différentes: interventions sur le patrimoine architectural lui-même, mise en place d'itinéraires thématiques dans le «musée en plein air » que constitue le centre antique (Vie dell'Arte, opération Decumani) ou encore institution de nombreux festivals et rendez-vous tels l'Estate a Napoli, Festival de théâtre, Napulanno, Mai des monuments qui demeure la manifestation-phare de l'offre touristique et culturelle de la ville ${ }^{4}$. Ces actions visent aussi bien une

I Le PAN $\left(6000 \mathrm{~m}^{2}\right)$, situé dans le Palais Roccella du XVIII s., a été inauguré le 26 mars 2005 comme centre multifonctionnel destiné à accueillir des expositions temporaires, des évènements culturels, et comme centre de documentation pour les arts visuels.

2 Le lanificio qui occupe, sur une surface de $600 \mathrm{~m}^{2}$, une partie du complexe conventuel annexé à l'église Santa Caterina a Formiello, tire son nom de l'usage qui en fut fait entre 1824 et 1860 par un noble napolitain transformant le lieu en le lanificio Sava, usage dont témoigne encore la cheminée qui domine le quartier de Porta Capuana. Voué à devenir une école élémentaire et fragilisé par le tremblement de terre de 1980, il est laissé à l'abandon durant 20 ans, avant d'être restructuré pour devenir un immense atelier de création artistique.

3 Quant à la définition de culture et de politiques culturelles, voir Grésillon B. (2004).

4 De la fin avril au début du mois de juin soit durant 5 à 6 week-ends, plus de soixante structures, monuments ou sites, sont ouverts gratuitement au public et accompagnés de programmes d'itinéraires thématiques et de manifestations culturelles. Pour une analyse approfondie de cette manifestation et de son évolution voir Palestino M.F. (2003) 
attractivité accrue de la métropole pour les touristes nationaux ou étrangers que sa redécouverte par les Napolitains eux-mêmes. Mais, hormis la valorisation du patrimoine artistique existant, le concept de ville d'art se décline de plus en plus du côté de la création d'équipements culturels de prestige. Le MADRe rejoint ainsi la liste des nouveaux musées méditerranéens comme le Centre andalou d'art contemporain de Séville, le Villa Croce à Gênes, le Mac à Marseille, le récent Maxxi conçu par Zaha Hadid inauguré à Rome en novembre 2009 et surtout le Guggenheim à Bilbao.

- La requalification du centre historique et la redéfinition de son rôle face à l'affirmation de nouvelles centralités est associée à la fonction culturelle qui figure comme pièce maîtresse du renouvellement urbain de Naples. Il s'agit d'un espace enjeu dans une ville en crise comme le sont bien d'autres villes européennes affaiblies par la désindustrialisation, de Glasgow à Marseille, mais à Naples son extension en surface, la diversité et la richesse de son patrimoine architectural, reconnu par l'Unesco en juillet 1995, lui confèrent un fort potentiel de développement. Les politiques urbaines ont misé ainsi d'emblée sur cet espace porteur d'un capital symbolique vivace quoique malmené, pour recomposer la ville sur elle-même, en véhiculer une image positive et reconquérir la confiance des habitants dans les institutions urbaines, fortement altérée par de nombreuses années de malgoverno.

- Enfin, le marketing du lieu qui vise à effacer « les iconographies négatives du déclin » (PALESTINO, 2007). La campagne publicitaire qui a accompagné pas à pas les ouvertures successives des stations de métro participe de ces stratégies de marketing urbain. Le recours à l'art contemporain, sous différentes formes, la création de nouveaux objets architecturaux portant la griffe d'artistes de renommée mondiale font partie d'une vaste entreprise de séduction. Là encore, le processus ne présente rien de vraiment original. Il s'agit d'une recette bien huilée à l'échelle internationale et ce n'est pas une première à Naples non plus, mais le rythme s'accélère après 2000 avec les stations signées par de prestigieux architecte - Gae Aulenti pour la Place Dante, Dominique Perrault pour la place Garibaldi - ou dans le cadre d'opérations d'envergure dans la banlieue de Naples avec Renzo Piano pour la réalisation à Nola, du Vulcano Buono, immense centre associant commerces, hôtellerie, loisirs, inauguré en 2008.

Toutefois à Naples, en raison de la pénurie de financements au début de la décennie 1990 - la ville est déclarée en faillite sur fond de crise de solidarité Nord-Sud et de fin de l'intervention extraordinaire - , le renouvellement de l'image s'est appuyé dans un premier temps sur des bricolages et des montages astucieux qui ont drainé davantage les synergies politiques et sociales que les ressources financières. La période qui s'ouvre au tournant du millénaire, marque en revanche un infléchissement de ces choix qui rejoignent avec quelque retard les standards de la promotion globale, dans un contexte de circulation de plus en plus rapide des modèles urbains à l'échelle mondiale, bref le syndrome Guggenheim au risque de la standardisation des identités urbaines. À Naples comme ailleurs, la relance de la « ville d'art » par le patrimoine historico-artistique et la culture, a donc au départ pour objectifs de soutenir la compétition internationale entre les métropoles, de proposer de nouvelles opportunités touristiques conciliables avec les pratiques citadines, de former un consensus social autour de la culture, en l'occurrence ici de l'art contemporain.

À l'occasion de l'inauguration du musée, le gouverneur de la région déclarait: " Aujourd'hui, nous avons le MADRe qui est un grand musée international, de la Région, de la ville et du centre de Naples. ». De quelle façon le musée est-il alors susceptible de conjuguer ces différentes échelles, de participer à ces dynamiques de développement local qui se veulent à la fois tournées vers l'extérieur, vers un affichage international, et endogènes, avec une insertion réussie dans le territoire urbain?

\section{2 - Musée MADRe, une insertion paradoxale?}

\section{I - Le MADRe, contrepoint aux stations de l'art}

L'avènement du MADRe complète en beauté le dispositif d'affirmation de Naples comme ville d'art à tous les effets. Il relève toutefois d'une logique différente de celle qui a prévalu pour les stations de métro, prenant en apparence le contrepied du parti pris adopté pour ces dernières. Les stations de l'art, dans la filiation de l'« art direct » des constructivistes attaché à la production d'éléments de la vie quotidienne, témoignent d'une volonté de « civiliser le mouvement ${ }^{5}$ en équipant des territoires métropolitains d'un réseau de transport public alliant l'esthétique et le fonctionnel, les transports et la culture. Elles renvoient aussi à l'émancipation des formes artistiques par rapport aux espaces des musées, à « l'expression d'un lien territorial ». L'irruption des stations de la ligne 1 dans le paysage urbain joue sur la création de nouveaux espaces, sur la mise en scène des espaces publics, autour des places notamment, ainsi que sur la volonté de conférer une identité à des espaces souvent considérés comme non-lieux ${ }^{6}$ et perçus comme anonymes. De ce point de vue, si Naples se situe dans le sillage de nombreuses autres expériences dans ce domaine ${ }^{7}$, méditerranéennes ou non, elle peut s'enorgueillir de réalisations très abouties, fondées sur l'articulation entre le réaménagement des places aux abords des stations et les formes muséales souterraines.

Là où le métro, espace ouvert, joue sur le rythme et la répétition des stations, tout en déclinant leurs différences, là où on peut déceler un effort de dialogue avec les espaces urbains environnants, le MADre s'affiche au contraire comme expérience concentrée sur un lieu unique, fermé, monumental. La naissance du MADRe repose sur la transformation du Palais Donnaregina, ancien bâtiment conventuel détenu par le Banco di Napoli ${ }^{8}$ et précédemment occupé par le siège de l'Inspection académique, ainsi que sur sa réaffectation à

5 Expression de François Béguin.

6 In Metrò-polis, catalogue de l'exposition (Castel dell'Ovo, 23 Décembre 2006-20 février 2007).

7 Stations de Norman Foster à Bilbao, le Roc aux sorciers de Jean-Luc Moulène à l'entrée de la station Gerland à Lyon, station Palais Royal entrée rue Colette à Paris, nouveau métro d'Athènes.

8 L'édifice, résultat de stratifications historiques qui ont concerné tout le quartier entre la fin du XVI et le XVII ${ }^{\mathrm{e}}$ siècle, est constitué de deux parties : l'une construite vers 1862, l'autre implantée probablement au XVII et profondément transformée au XIX ${ }^{\mathrm{e}}$ siècle. Lui est associée également la chapelle Donnaregina adjacente. 
un usage muséal plus classique, consacré à l'art contemporain, même si ont été adjointes d'autres fonctions culturelles et récréatives. Sur les $8000 \mathrm{~m}^{2}$ qu'il occupe, 4500 sont consacrés à l'exposition d'oeuvres d'art. Le lieu est doublement voué à devenir le temple méridional de l'art contemporain: en tant qu'écrin somptueusement réaménagé par l'architecte portugais Alvaro Siza y Vieira et par la valeur des œuvres qu'il abrite. Le principe est de faire dialoguer sur trois niveaux différents langages artistiques, issus d'horizons géographiques divers mais laissant une place de choix aux artistes liés à la ville, depuis les salles monographiques inédites, appropriées in situ par les artistes, aux collections « permanentes » et aux salles d'exposition temporaire.

Cette insertion répond à une volonté de reconquête interne des territoires du centre historique, de construction d'une centralité culturelle, bien connectée au réseau de transport grâce à la proximité des stations Musée et Place Cavour. De ce point de vue, le MADre diffère de nombreux équipements culturels liés à de vastes opérations de régénération urbaine sur des friches plus excentrées - Guggenheim à Bilbao ou Friche de la Belle de Mai à Marseille.

Le territoire d'insertion, en revanche, est clairement du côté de la marginalité sur les plans économique et social, comme en témoignent les indicateurs de chômage qui avoisinent les $36 \%$ dans le quartier populaire de San Lorenzo, les faibles taux de scolarisation $-40 \%$ des habitants ont au mieux terminé l'école élémentaire - , ou encore la part des immigrés dans la population. Une situation qui en fait un lieu quelque peu paradoxal, marqué par une contradiction non résolue entre ouverture et ruptures.

\section{2 - Un lieu de prestige, entre ouvertures et ruptures}

Par bien des aspects, le MADre est un lieu tourné vers l'extérieur, ouvert à l'échelle nationale et internationale. La centaine d'oeuvres de la collection permanente vient d'artistes décisifs dans la création contemporaine mondiale depuis quarante ans et dépasse largement le cadre italien, retraçant un dialogue artistique entre Europe et États-Unis. En outre, les œuvres du deuxième étage, prêtées à temps indéterminé par les galeries napolitaines et italiennes ainsi que par de très nombreuses galeries étrangères, témoignent d'une insertion réussie dans des réseaux de relations culturelles à l'échelle européenne et renforcée au fil des accords passés avec de grands musées italiens et étrangers tels que la Tate Gallery de Londres ou le musée Ludwig de Cologne, à l'occasion des expositions temporaires.

Le lieu est ouvert aussi à l'échelle métropolitaine selon un fonctionnement en réseau et en écho avec les autres espaces culturels qui lui sont associés lors de grands évènements culturels. On peut citer par exemple le partenariat avec le musée Capodimonte pour l'exposition sur le baroque, programmée pour décembre 2009, avec le Castel Sant-Elmo et les installations de la place du Plébiscite et, audelà des limites de la commune, avec la Reggia de Caserte. L'ouverture passe aussi par les échanges avec d'autres formes et registres artistiques comme la bande dessinée, le théâtre et la musique, jazz notamment.

Parallèlement, le musée draine une proportion croissante de touristes en liaison avec les itinéraires de mieux

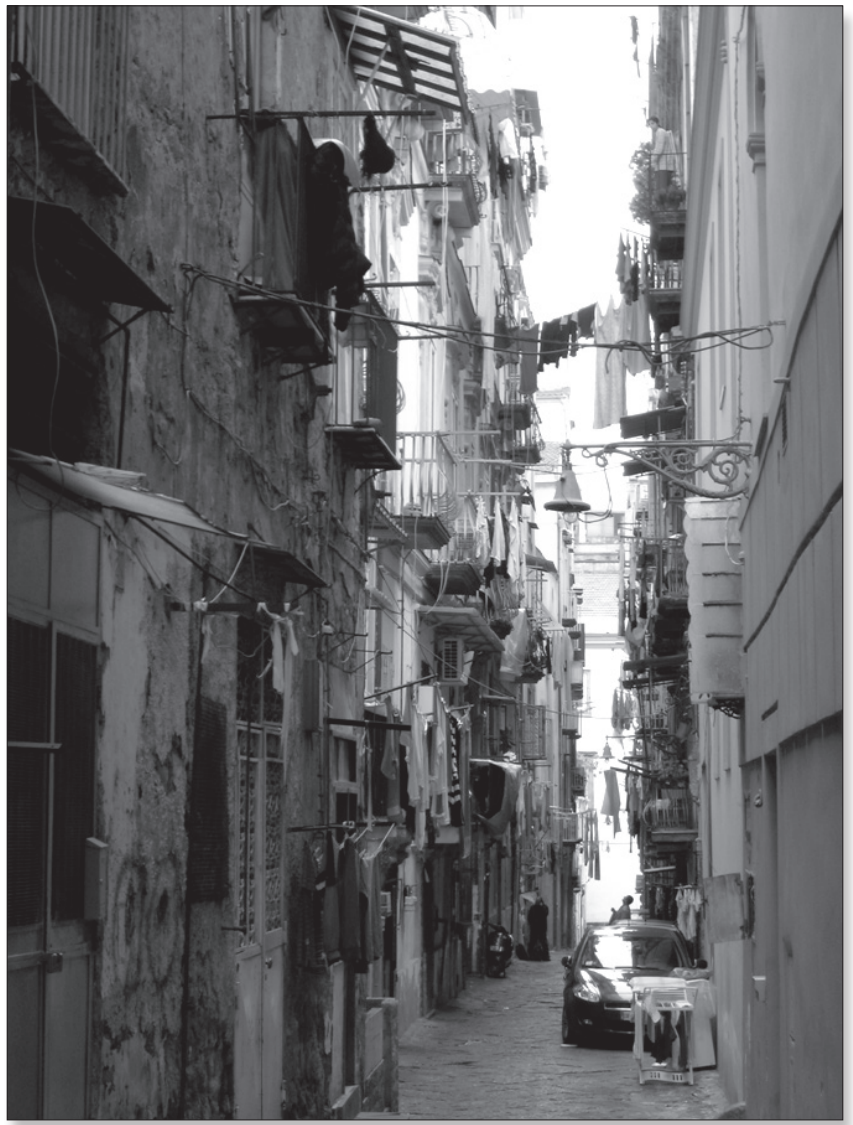

Photo 1 - Via Loffredi, façade extérieure du musée à droite. Cliché P. Froment.

en mieux balisés du centre historique et avec la proximité du musée archéologique et de la cathédrale. Depuis l'ouverture, le nombre de visiteurs a significativement augmenté : les entrées passent de 53000 en 2006 à 75000 en 2008, mais restent encore très limitées par comparaison avec le musée d'art moderne Georges Pompidou qui accueille 1,1 M de visiteurs chaque année ou le Guggenheim de Bilbao avec près de $1 \mathrm{M}$ actuellement.

Le musée MADRe, en tant qu'équipement polarisant, peut-il alors jouer un rôle moteur dans la transformation des territoires environnants?

La réponse ne va guère de soi car le MADre est incontestablement un lieu que l'on peut lire aussi sous l'angle de la rupture. Les lignes de faille ne sont pas toutefois à rechercher dans la forme architecturale externe. À la différence des Guggenheim de New York et de Bilbao ou du centre Georges Pompidou à Paris, dont les bâtiments peuvent être considérés comme de véritables sculptures contemporaines, œuvres d'art audacieuses et autonomes, le parti pris d'Alvaro Siza a misé sur le contraste voulu entre le patrimoine architectural de la vieille ville et les langages de l'art contemporain, entre la conservation de l'ancien bâtiment et le réaménagement intérieur. Si l'on peut parler de rupture visuelle, celle-ci se condense autour de deux éléments : d'une part, le soin et l'aspect florissant de l'édifice rénové qui contraste vivement avec l'habitat très dégradé des rues adjacentes comme la rue Loffredi (photo 1) et d'autre part, la valeur des oeuvres nichées dans cet écrin sophistiqué, qui renvoie en négatif au dépouillement économique et à la précarité sociale de la population résidente. L'immersion « physique » de ce lieu dans le quartier populaire de San 


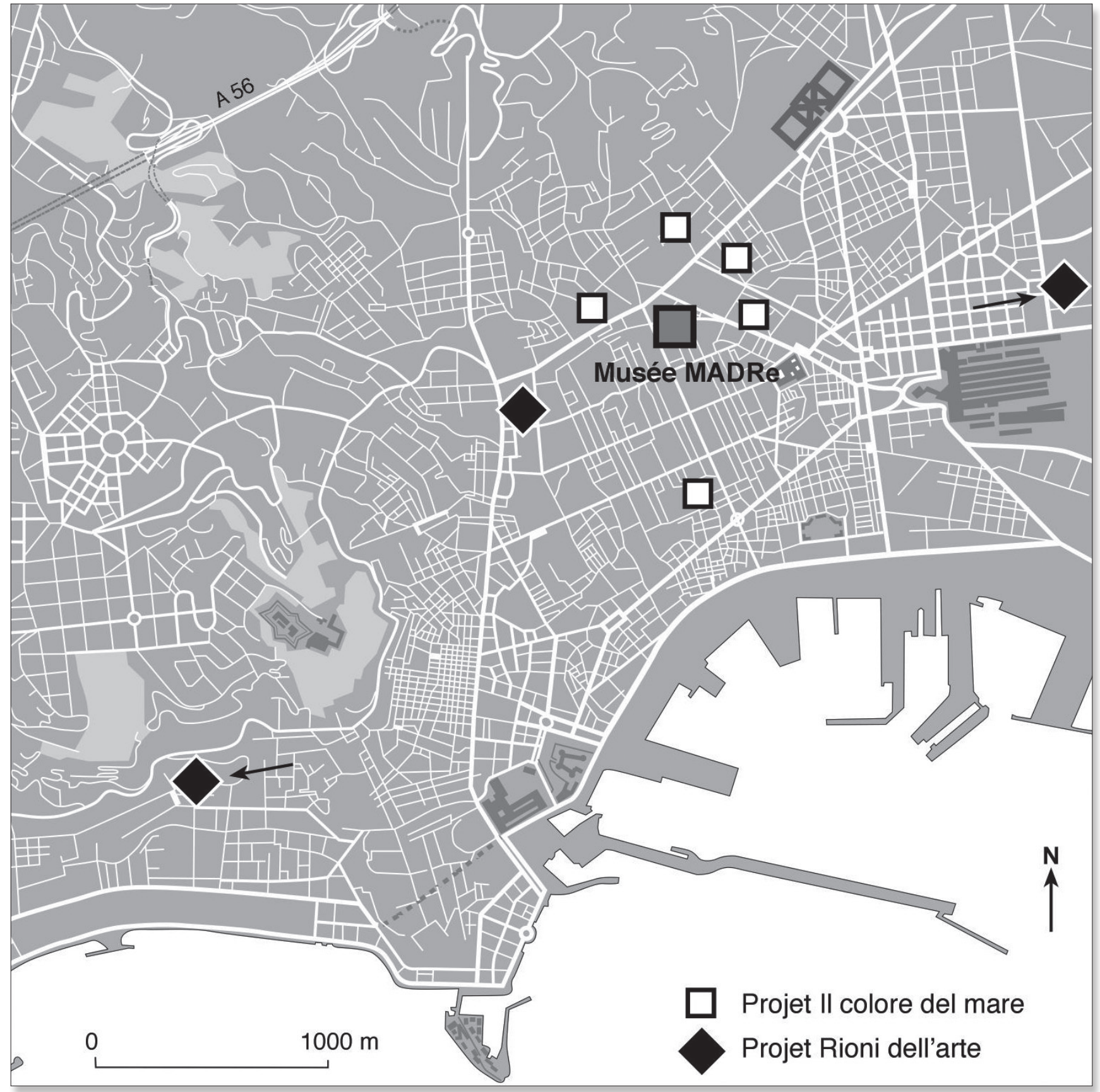

Fig. 2 - Territoires et éducation à l'art.

Lorenzo, créant une promiscuité inattendue, non seulement n'abolit pas la distance culturelle mais semble au contraire la souligner. « Dans la mesure où l'accès à l'art est davantage une question de niveau d'éducation que de proximité de l'équipement, l'augmentation de l'offre culturelle ne peut donc pas résoudre les questions d'accès à l'art » (FOUR, 1995). Une distance qui se doit par conséquent d'être conjurée par les tentatives récentes de rapprochement, de comblement du fossé. Les objectifs sont doubles: amener d'une part les populations des quartiers populaires centraux à fréquenter le musée dans une perspective de démocratisation de l'art contemporain, d'autre part permettre à terme une transformation du quartier par l'installation de nouvelles populations, conformément à une quête institutionnelle ambiguë de mixité sociale, escomptée dès le départ dans les différents secteurs revitalisés du centre historique.

L'objectif de requalification du quartier, et donc d'ouverture au territoire environnant se développe depuis un an autour d'initiatives d'éducation à l'art, une vocation inhérente à la définition même de musée?. Le projet Une politique de l'art pour les jeunes, qui a démarré en octobre 2008, est promu par la Fondation Donnaregina, avec la contribution de l'Assessorat Régional aux Politiques Sociales et pour la jeunesse et du Banco di Napoli pour $l$ 'assistance à l'enfance. Il concerne en priorité les territoires limitrophes du musée, circonscrit dans un rayon de 500 mètres, mais touche également des espaces plus

9 La dimension sociale des musées, ouvrant une perspective d'engagement de la profession, est fixée par l'Icom en 1972, et cette mission est reprise par la plupart des définitions nationales ex l'Association Américaine des Musées n'accrédite de musée que si l'institution est essentiellement éducative 
éloignés ${ }^{10}$ (fig. 2). L'idée de musée obligatoire, d'art public et populaire, développé par Achille Bonito Oliva", à propos des stations de métro, rejoint ici celle du musée accessible, visant à amener les habitants des quartiers populaires au langage de l'art contemporain, en partant de l'éducation des jeunes et d'une implication régulière de leurs parents. Ces initiatives, organisées sous forme d'ateliers, s'adressent d'une part aux établissements scolaires, écoles et collèges, selon un rythme de trois matinées par semaine sur une durée de trois mois, et d'autre part aux jeunes pris en charge par différentes associations - Associazione Quartieri Spagnoli, Manitese, La casa dello scugnizzo - dans l'après-midi. Elles ont réuni au total sur la saison 2008-2009, 5 établissements, 500 élèves, 35 jeunes venus des associations, 22 enseignants et responsables d'association, 8 opérateurs culturels. Les expositions au sein du musée qui ont conclu les deux cycles - La couleur de la mer en décembre et Les Quartiers de l'art en juin -, outre la valorisation et la communication du travail réalisé, visent également une ouverture du musée aux familles des enfants ayant participé aux projets. Toutefois la route risque bien d'être longue. Les quelques enquêtes effectuées à titre de test montrent que la population résidente, tout en connaissant la présence du musée, n'en a jamais dépassé le seuil.

Il est bien sûr trop tôt - quatre ans seulement - pour dresser un bilan des retombées de l'implantation du musée sur les territoires voisins. Et de plus, on ne peut guère raisonner dans le cas du MADre, à l'aune des grands projets de régénération urbaine qui seraient davantage le fait d'autres espaces de la cité comme la zone portuaire ou le site de Bagnoli dont le destin reste encore en suspens. Cependant on peut déceler quelques signes liés à une fréquentation accrue de la zone, du côté plus passager de Via Foria et de via Duomo - un café modernisé, le changement de deux boutiques dans le voisinage immédiat du MADre -, alors que la restructuration en cours de trois immeubles donnant sur la rue Settembrini, peut signifier à plus long terme un changement du profil de la population à l'échelle de quelques îlots.

\section{3 - Le musée Madre comme symbole de la fermeture du dialogue entre institu- tions et forces vives de la ville}

L'ouverture des stations de l'art, puis celle du MADre procèdent de logiques voisines sous l'angle cette fois de la gouvernance. Elles apparaissent en effet comme deux étapes cruciales dans un processus de « rigidification » croissante des pratiques de gouvernement de la ville et d'isolement de l'acteur public, dont les politiques culturelles dans leur ensemble, et en particulier celles destinées à l'art contemporain, ne seraient finalement que l'un des symptômes.

\section{I - Espaces pratiqués, espaces contestés}

Parmi les réalisations liées à l'art contemporain, les stations de la ligne 1, constituent sans aucun doute l'action la plus remarquable mise en œuvre par la municipalité de Naples. L'achèvement rapide des six stations centrales a eu une incidence réelle sur la ville, modifiant les habitudes, opérant un rapprochement entre les périphéries les plus déshéritées et le centre, influant fortement aussi sur le marché immobilier, avec un doublement de la valeur des immeubles dans la zone de Salvatore Rosa par exemple. Il semble aussi que le concept de station de l'art ait, peu ou prou, atteint son objectif en tant qu'outil de marketing urbain touchant à la fois un public externe à la ville et la population napolitaine. Mis en scène par une solide campagne publicitaire, à visées également électorales, l'avancement du métro a fait parler de lui à l'extérieur que ce soit dans les colonnes du Times ou dans les salles de la Biennale de Venise en 2002, emportant du même coup une adhésion mesurée de la population. Il est certes difficile d'en déduire une véritable appropriation par les usagers, en l'absence d'études précises: les effets de ce « musée obligatoire » restent à cet égard à interroger et à mesurer plus finement. À l'intérieur comme à l'extérieur, les stations de la ligne 1 du métro sont rutilantes, préservées, respectées, phénomène assez rare pour être relevé.

À l'échelle de la ville, le projet fait aussi école. Si au départ les stations de l'art ne devaient concerner que quelques points névralgiques, le projet s'est par la suite étendu et elles concernent également d'autres lignes du réseau à l'échelle régionale: la Circumvesuviana et la Cumana, créant de nouvelles expectatives de la part de quartiers périphériques en crise comme celui de Scampia, dont la revendication prend une dimension plus clairement identitaire autour de la figure de Felice Pignataro dont l'activité artistique s'est durablement ancrée dans le quartier'2.

Il en va tout autrement du musée MADre, devenu très rapidement un espace contesté, faisant l'objet de vives polémiques, en tant que lieu destiné à un usage essentiellement touristique et vécu trop exclusivement par un public externe à la ville ou réservé à une élite napolitaine.

Parmi les formes très variées de cette contestation - largement rapportées dans les colonnes de la presse locale à propos notamment de la légitimité de certaines activités de discothèque -, nous avons retenu deux moments, correspondant à deux contre-expositions qui reflètent les questionnements relatifs aux lieux et aux politiques de l'art contemporain, à Naples. Le premier a lieu en février 2007, un an et demi après l'ouverture du MADre, à l'occasion du vernissage de l'exposition de Rachel Whiteread et Marisa Merts. Cette manifestation culturelle se voit doublée par l'inauguration en plein air d'une contre-exposition intitulée

par nature: $88 \%$ des musées des ÉtatsUnis fournissent des programmes éducatifs centrés sur l'art, l'histoire, les sciences; il en va de même pour la loi française de janvier 2002 sur la définition «musée de France » (D. Poulot, 2009).

10 Le deuxième cycle a impliqué trois aires urbaines et trois municipalités (S Pietro a Patierno, Stella-San Carlo et il Casale). Le thème majeur de l'atelier porte sur l'appartenance au territoire et sur le développement de l'attention pour le « bien commun ». Il a concerné 15 classes, 15 artistes, 12 experts, plus de 100 enseignants et 300 enfants ainsi que leurs parents et a été réalisé en partenariat avec Aporema Onlus, structure à but non lucratif dont l'objectif est de favoriser la diffusion des langages artistiques.

II Achille Bonito Oliva est critique d'art et professeur d'histoire d'art contemporain à l'université La Sapienza à Rome, cf. Metropolis cité ci-dessus.

12 Cf. Palestino M.F. et également le site [www.fuoricentroscampia.it]. 
Diniego, ou Dénégation, Refus, dont les murs de la rue Loffredi qui longe une façade latérale du MADre, conservent encore aujourd'hui quelques traces pâlies. La contestation, à l'initiative de deux artistes de rue suivis par d'autres, joue sur un double registre associant l'économique et le politique. Au-delà d'une mise en cause du consumérisme, il s'agit davantage de la contestation d'un art institutionnalisé et d'un espace culturel conçu comme « une cathédrale dans un désert, des vernissages d'élites, des griffes étrangères, sans aucun contact avec la ville ${ }^{13} »$. Cette mise en cause prend la forme de pastiches à partir des dépliants officiels du MADre dévoyant leur sens par des anagrammes et des jeux de mots; ainsi, MADRE devient MERDA et les logos Regione Campania sont remplacés par Regione Camorra. En avril 2009, la contestation de l'exposition Urban Superstar Show consacrée à l'urban art, se déploie autour du musée le jour de l'inauguration, sur un mode davantage pacifique et bon enfant, donnant lieu à des performances en extérieur. L'une d'entre elles en particulier, signée par le collectif Satoboy, met en scène un grand nombre de poupons installés à même sol, symbolisant les enfants non reconnus et oubliés - à savoir les artistes de rue napolitains - par cette Madre Snaturata, textuellement Mère dénaturée, qui n'a d'yeux que pour les horizons lointains. De fait, l'exposition ambitieuse organisée par la Galerie romaine Mondodop qui s'ouvre intra-muros n'a laissé aucune place aux figures méridionales du street art dont certaines sont pourtant reconnues à l'échelle de l'Italie. « Poser un jalon qui fait de l'empreinte sur le territoire le fondement même de son mode d'expression, sans dialoguer avec le territoire en question, sans voir qui déjà y a laissé des traces, est un signal d'alarme.»> ${ }^{14}$. Une occasion manquée en quelque sorte.

Sous des formes diverses, les deux évènements posent en fait la question du positionnement ambigu du MADre. S'il doit répondre à l'ambition des édiles locaux d'en faire un grand musée d'art contemporain sur l'échiquier international et national, il n'en est pas moins destiné à devenir une référence pour la Campanie et pour l'ensemble du Sud de l'Italie, or les orientations artistiques laissent paradoxalement de côté le patrimoine de son hinterland culturel « naturel ». La solitude du MADre, tournant le dos à son territoire, à différentes échelles, apparaît comme le symbole d'un blocage, d'une fermeture du dialogue entre les différents acteurs de la métropole, entre les instances du gouvernement local et les forces vives de la ville.

\section{2 - Glissements}

Le passage des premières initiatives culturelles, notamment celle de Porte Aperte aux projets plus ambitieux du métro et puis du MADre, témoigne d'un glissement dans les stratégies de renouvellement urbain et dans les pratiques de gouvernance. On passe en effet de logiques ouvertes et participatives de type bottom up qui dominent dans les cinq premières années de la Municipalité Bassolino à des logiques top down qui l'emportent clairement au tournant du millénaire. Elles sont marquées par un déficit démocratique croissant et une faible association des citoyens à l'élaboration des projets, évolution qui va de pair avec une affirmation des logiques inside out, misant sur l'image à l'extérieur au détriment du dialogue avec le territoire.

Le projet des stations artistiques, impulsé par la municipalité, à travers la société Métropolitana di Napoli, relève déjà d'une « une action aristocratique », pour reprendre les mots de Maria Federica Palestino. Il est porté par la volonté, l'enthousiasme et les décisions d'un nombre très restreint d'acteurs très liés entre eux, issus du personnel politique en place ou du monde de l'art. Parmi eux le maire-adjoint Vincente Marone, le président de la société Metronapoli, l'architecte Alessandro Mendini, le critique d'art Achille Bonito Oliva et les principaux galeristes napolitains.. Mais le groupe se restreint encore avec le MADre, dont le projet relève au départ d'une décision régionale. Toutefois si l'échelle des initiatives change entre les deux projets, on ne peut manquer de relever une forte continuité du côté des acteurs impliqués: Antonio Bassolino, maire de Naples de 1993 à 2000, devient alors gouverneur de la région Campanie, conservant une partie son entourage dans le secteur culturel. La création en 2004 de la Fondation Donnaregina, chargée de la promotion et de la gestion de l'art contemporain, par la Région Campanie, témoigne de la concentration des décisions dans un cercle de plus en plus fermé d'initiés en matière de politiques culturelles. Elle est présidée par Antonio Bassolino lui-même, dirigée par Edoardo Cicelyn, déjà conseiller culturel pour la Région de 2002 à 2005 et nommé à cette date directeur du MADre, et compte dans le Comité scientifique Achille Bonito Oliva, devenu une figure incontournable dans les affaires artistiques de la ville. Augusto de Luca, photographe et co-instigateur de la contre-manifestation de 2009, parle à leur propos de « barons du pouvoir de l'art contemporain à Naples ».

Le musée est largement financé par les fonds de l'Union européenne via la Région et notamment le Programme Opérationnel Régional de la Campanie pour les périodes 2000-2006 et 2007-2013. Outre le financement de la restructuration du bâtiment (45 M€) et l'acquisition des douze œuvres du premier étage ( $3 \mathrm{M} €$ ), les fonds alloués pour les dépenses s'élèvent à environ 6,5 M€ par an, partagés entre la gestion courante et les activités artistiques proprement dites. Le musée bénéficie ainsi de fonds substantiels en comparaison des autres structures ou manifestations artistiques de la ville.

Les pratiques de gouvernement dans le domaine de l'art contemporain traduisent de fait un déficit de transparence et de contrôle démocratique tout autant que les difficultés croissantes des institutions locales à stabiliser le consensus établi dans les années 90, à dialoguer avec les élites entrepreneuriales et les autres acteurs de la société civile. Le problème vient essentiellement des modalités d'accès

13 Cf. article de Paolo Mossetti (14 mars 2007, site www.ilrichiamo.org). Pour le directeur du Madre, la contestation sonne faux dans la mesure où elle n'est pas un défi artistique mais un défi politique et économique: sa proposition d'ouvrir et de consacrer à ces artistes de rue un espace au sein du musée n'a pas abouti du fait des artistes eux-mêmes (entretien du avril 2009).

14 Borriello L. directeur de Inward, propos rapportés in corrieredelmezzogiorno.corriere.it « Mostra senza gli artisti del Sud»; aussi AgoraVox Italia, mercredi 22 avril 2009. 
aux diverses fonctions et missions qui n'ont pas fait l'objet de concours et d'appels d'offre publics. Cela concerne aussi bien la sélection des artistes retenus pour la réalisation des stations de métro que l'attribution de certains postes clés, comme par exemple celui de directeur du MADre. L'octroi des différentes responsabilités procède le plus souvent de la « volonté du prince », accréditée dans certains cas par l'intervention d'un « arbitre » extérieur, plutôt que de procédures vraiment démocratiques.

Les partenariats sont élaborés de façon prédominante selon une dynamique interinstitutionnelle, impliquant peu les acteurs privés dans les décisions et isolant l'acteur public, et demeurent dans l'ensemble limités à une dimension de gestion. Ainsi le MADre, comme d'ailleurs de nombreux sites du patrimoine régional, est géré par la Scabec SpA (Società campana per i Beni Culturali), une société de services mixte créée en 2002. Elle est détenue à $51 \%$ par la Région et $49 \%$ par le privé représenté par la Rti, association de 12 entreprises dont Mondadori-Electa et Prc, société leader en Italie dans les services de billetterie.

\section{Conclusion}

En termes de communication et de dialogue avec la société civile, l'ouverture des stations de l'art s'est largement appuyée sur une stratégie de marketing interne et externe, qui visait davantage la consolidation d'un consensus que l'information proprement dite des citadins. Mais fût-ce de façon passive, ces derniers ont pu suivre et participer à cette mise en scène de leur ville qui devenait de nouveau présentable, voire même enviable par les autres métropoles européennes. La création du musée MADRe n'a donné lieu en revanche à aucune tentative de ce genre, entérinant ainsi une coupure déjà manifeste entre les acteurs des politiques urbaines et la société civile, et niant toute implication possible de cette dernière.

Ainsi les projets les plus récents en matière de culture, s'ils mobilisent certains ingrédients nécessaires de la « ville entrepreneuriale »- nouveaux espaces et édifices, artistes phares, marketing urbain - et fournissent des pièces de qualité dans le dispositif culturel et urbanistique, se détournent « d'une véritable stratégie de la citoyenneté » pourtant au fondement des politiques culturelles. Le MADRe en particulier reflète l'ambiguïté du discours politique en matière de modalités de participation des populations, témoignant plutôt d'une volonté factice de légitimation sociale des projets, intervenant a posteriori et imposée d'en haut. Aussi les professions de foi réitérées par les acteurs politiques en place, relèvent-elles plus de l'invocation que d'une réelle stratégie à l'égard des secteurs socialement les plus dégradés. La faiblesse du contenu social des interventions, en liaison avec les nouvelles structures culturelles, demeure en fait une réponse standardisée et mal ajustée aux problèmes posés. En fin de compte, le MADre qui, malgré des qualités et des potentialités indéniables, ne possède ni l'aura ni la puissance d'un Guggenheim, reste à ce jour une figure extra-territoriale, un objet tourné sur lui-même, un relais de diffusion d'une culture institutionnelle, mal intégré au tissu urbain environnant.

À Rachel Rodrigues-Malta, en souvenir de nos parcours napolitains.

\section{Bibliographie}

BARRE F., (2002), « Contours et alentours » in Charbonneaux A.-M., Hillaire N. (dir.), Euvre et lieu, Paris, Flammarion, p. 5-17

Cahiers de l'IAURIF, (2007), « Grands projets urbains en Europe », mars, n 146.

BÉGUIN F., (1993), « Civiliser le mouvement », Appel d’offres « Espaces publics » DAU , Plan construction.

BENIT Cl., GRÉSILLON B., (2004), « La réhabilitation de la manufacture de la Belle de Mai » in La lettre électronique de MIP-Provence, $\mathrm{n}^{\circ}$ 7, septembre, p. 1-6.

CASCETTA E. dir., (2005), La sfida dei trasporti in Campania. Mobilità e sviluppo sostenibile, Electa, Napoli.

FLORIDA R., (2002), The Rise of the Creative Class, And How It's Transforming Work, Leisure, Community and Everyday Life, New York, Basic Books, 404 p.

FOUR P.A., (1995), Intervention publique et art contemporain: invention, insertion, et acquisitions des fonds régionaux d'art contemporain, thèse de doctorat de l'Institut d'études politiques de Paris, 585 p.

FROMENT P., (2003a), «Les espaces publics dans les politiques patrimoniales à Naples : place du Plébiscite et place Dante » in Patrimoine et politiques urbaines en Méditerranée. Revue Rives nord-méditerranéennes, nº 16, p. 99-118.

- (2003b), « La renaissance de Naples - Réussites et ambiguïtés » in Les cahiers espaces, n 78 , juillet, Tourisme urbain, p. 36-46. 
GILABERT T., (2004), La géographie de l'art contemporain en France, thèse de doctorat, université de Nantes.

GRÉSILLON B., (2002), Berlin, Métropole culturelle, Belin, coll. Mappemonde, 351 p.

LAGNIER S., (2001), Sculpture et espace urbain en France: histoire de l'instauration dialogue 1951-1992, L'Harmattan, Paris, coll. «Logiques sociales», 291 p.

MASBOUNGi A. (dir.), (2004), « Penser la ville par l'art contemporain », in Projet urbain Editions de la Villette, p. $107-112$.

PutMan, J., (2002), Le Musée comme médium dans l'art contemporain, Thames \& Hudson, Paris, 280 p.

PALESTino M.F., (2007), « Fra innovazione e restaurazione. Dieci anni di pianificazione culturale », in Belli A. (dir.), Non è cosi facile. Politiche urbane a Napoli a cavallo del secolo, Franco Angeli.

- (2003), MiraNapoli la costruzione dell'immagine urbana negli anni'90, CLEAN Edizioni, Naples.

Poulot D., (2009), Musée et muséologie, La Découverte, coll. Repères, 125 p.

RODRIGUES-MALTA R., (1999), « Villes d’Espagne en régénération urbaine. Les exemples de Barcelone, Bilbao et Madrid » in Les annales de géographie, ${ }^{\circ}$ 608, juillet-août, p. 397-419.

- (2001a), « Régénération urbaine: variations sud-européennes » in L'Information géographique, vol. 65, n 4, p.321-339.

- (2001b), « Esthétique des infrastructures et régénération urbaine » in Prelorenzo Cl., Rouillard D. (dir.), Mobilité et esthétique, deux dimensions des infrastructures territoriales, L'Harmattan, $142 \mathrm{p}$.

SCOTT A. J., (1999), « L'économie culturelle des villes », Géographie, économie et société, vol. 1, n 1, p. 25-47. 